\title{
Extended Approach of ANFIS in Cascade Control
}

\author{
Mahardhika Pratama, Samsul Rajab, and Er. Meng Joo
}

\begin{abstract}
-this paper discusses extended approaches of Adaptive Network Based Fuzzy Inference System (ANFIS) using Self Tuning Regulator (STR) via Fuzzy logic to be implemented in cascade control. Plant used is Pressure RIG 38-714 which supports cascade configuration. The controlled variable in the outer loop is pressure, in the inner loop's flow. The proposed method's used to improve the performance of ANFIS which has been commonly implemented to accomplish control task. Comparison has been conducted between ANFIS using STR and without STR, from experiment could be concluded that controller ANFIS using STR acquired better performance than only used ANFIS. It's derived from the transient response of those. For ANFIS using STR is obtained rise time and settling time are $9 \mathrm{~ms}$ and $12 \mathrm{~ms}$ respectively. In the other hand, ANFIS without STR resulted $21 \mathrm{~ms}$ and $29 \mathrm{~ms}$ for rise time and settling time respectively.
\end{abstract}

Index Terms-ANFIS, Cascade Control, Self Tuning Regulator.

\section{INTRODUCTION}

Cascade control's one of example in the complex architecture of control system. Indeed, it has two control loops, one's used to control main variable (outer loop) and another's used to reject the disturbance (inner loop). An advantage of this structure's if there's a disturbance on the inner loop might be immediately detected and undertaken self correcting, because process on the inner loop has to be much faster than on the outer loop, thereby such structure like that's also able to prevent a stability of the plant simultaneously. Although has an advantage, cascade control's relatively expensive due to utilizing more sensor. Because, there are two control variable to solve one main control variable.

Fuzzy logic invented by Zadeh has been widely used in the many applications. In the area of control engineering, fuzzy logic controller with many varieties and structures have been practically developed in the many areas such as: control, forecasting, identification, and etc. The strongest thing of fuzzy logic's can adopt intuition of human feeling mapped in the membership function of fuzzy logic. Such thing like that might replace on-off logic which only understands 2 values as $\mathrm{ON}$ and $\mathrm{OFF}$.

However, the weakness of fuzzy logic's it does not has a

Manscript received April 6, 2011; revised July 26, 2011

Mahardhika Pratama is with School of Electrical and Electronic Engineering, Nanyang Technological University, Singapore (Corresponding author to provide, e-mail: MAHARDHI1@e.ntu.edu.sg).

Samsul Rajab., is with Electrical Engineering Department, Sepuluh Nopember Institute of Technology, Indonesia (e-mail: samsulrajab@gmail. com).

Er Meng Joo is with the School of Electrical and Electronic Engineering, Nanyang Technological University, Singapore (e-mail: EMJER@ntu.edu. $\mathrm{sg})$ fix pattern to obtain the shape of membership function.

In the other hand, it's also difficult to establish the rules of fuzzy logic controller. Because of the above problems, those could be concluded that fuzzy logic controller's very dependable on the expert intuition.

A Neural Network (NN) technique's cited a lot of attention in the recent years basically adopting the principle of human brain. Many researches are conducted in the application of neural network. It just need to give training data with certain algorithm to be learned by NN. The learning phase determines a weight of link that connects each node.

In spite of very powerful, $\mathrm{NN}$ also has weaknesses. A NN is very dependent on the numbers of weights in the each links. It's time consuming to choose a suitable structure of NN corresponding to how many neurons and hidden layers to be utilized. A success of designing $\mathrm{NN}$ is determined not only in the performance of $\mathrm{NN}$ but also computational time needed by NN to accomplish a task, more neurons and hidden layers are more time to be taken in the learning phase.

Due to the above backgrounds, the basic idea to unite the NN and fuzzy logic controller is emerged by R.J.Jang to create ANFIS. Structure of Fuzzy logic is combined with learning principle of neural network, thus Problems can be eliminated including the problems in the obtaining the shape of membership function and suitable rule fuzzy logic controller. In the other hand, the problem to find structure of $\mathrm{NN}$ can be overcome because of fix structure of fuzzy logic structure.

The integrated neural-fuzzy system combines advantages of both NN and FIS. Application of both technologies are categorized into following four case [1] :

1) $\mathrm{NN}$ is used to automate the task of designing and fine tuning the membership functions of fuzzy systems.

2) Both fuzzy inference and neural network learning capabilities acting separately.

3) $\mathrm{NN}$ customizes the standard system according to each user preferences and individual needs.

Applications of ANFIS controller have been done in the several purposes such as: In [2], the author using ANFIS and self tuning to improve pitch and yaw of twin rotor. In [3] genetic algorithm (GA) was created based ANFIS, [4] ANFIS was used with PSO to control velocity control of DC motor, ANFIS as controller unmanned air vehicle was proposed in [5], [6] they developed ANFIS as stability controller of inverted pendulum, [7] ANFIS was compared with radial basis function neuro fuzzy with hybrid genetic and pattern search algorithm. Because of the significant contribution of ANFIS controller and cascade control, therefore it's important to enhance in the implementation of ANFIS.

This paper proposes controller ANFIS which combine with STR to be implemented in cascade control. The structure of the ANFIS is formed using five-layer fuzzy neural network. At the cascade structure ANFIS is employed 
in addition STR is used to complete the strength of ANFIS. The proposed method is used to act as primary controller in the outer loop, in the inner loop, pure PI controller is used. To know the abstraction of Plant model Auto Regressive with Exogenous input (ARX) is used via Pseudo Random Binary Sequence as input.

This paper's organized as follows: Section I gives the description and background of the research. Section II describes the basic theory of ANFIS, self tuning regulator, and cascade control. Plant modeling with dynamic identification via ARX is described in detail in Section III; in addition the experimental set up is also included. Section IV explains experimental result and discussion. Finally, some conclusions are given in the rest of this paper.

\section{LITERATURE REVIEW}

In this section is discussed the concepts of the ANFIS controller, self tuning regulator, and cascade control.

\section{A. Adaptive Network Based Fuzzy Inference System (ANFIS)}

As aforementioned, ANFIS is created from integration of fuzzy logic and neural network. This paper assumes that ANFIS under consideration has two inputs $x_{1}$ and $x_{2}$ and one output $f$. The structure of ANFIS is shown in Fig. 1. From if-then rules of Takagi and Sugeno's type can be defined as follows :

Rule 1: If $x_{1}$ is $A_{1}$ and $x_{2}$ is $B_{1}$, then

$$
f_{1}=p_{1} x_{1}+q_{1} x_{2}+r_{1}
$$

Rule 2: If $x_{1}$ is $A_{2}$ and $x_{2}$ is $B_{2}$, then

$$
f_{1}=p_{2} x_{1}+q_{2} x_{2}+r_{2}
$$

In which $A_{i}$ and $B_{i}$ are the membership functions of ANFIS (antecedent). $p_{i}, q_{i}, r_{i}$ are the consequent parameters. The node functions each layer describes as follows :

Layer 1: This layer's input layer that classify input of ANFIS controller into the fuzzy sets.

$$
\begin{aligned}
& O_{i}^{1}=\mu_{A_{i}}\left(x_{1}\right), i=1,2 \\
& O_{i}^{1}=\mu_{B_{i}}\left(x_{2}\right), i=3,4
\end{aligned}
$$

where $x$ is the input to node $i$, and $A_{i}$ is the linguistic label (fast, slow, etc).

Layer 2: After they are laid in the MF of ANFIS, AND operator is used before to be an input of next layer. Every node in this layer is a circle node with labeled $\Pi$. It's means that every incoming signal should multiplies and sends the product out.

$$
O_{i}^{2}=w_{i}=\mu_{A_{i}}\left(x_{1}\right) \cdot \mu_{B_{i}}\left(x_{2}\right)
$$

Layer 3: Every node in this layer a circle node labeled $N$. Input on this layer have to be normalized with calculating.

$$
O_{i}^{3}=\bar{w}_{i}=\frac{w_{i}}{w_{1}+w_{2}}, i=1,2
$$

Layer 4: The input from previous layer's multiplied with output parameters determined from learning process. Node function in this layer as follows:

$$
O_{i}^{4}=\bar{w}_{i} \cdot z_{i}=\bar{w}_{i} \cdot\left(p_{i} x_{1}+q_{i} x_{2}+r_{i}\right) i=1,2
$$

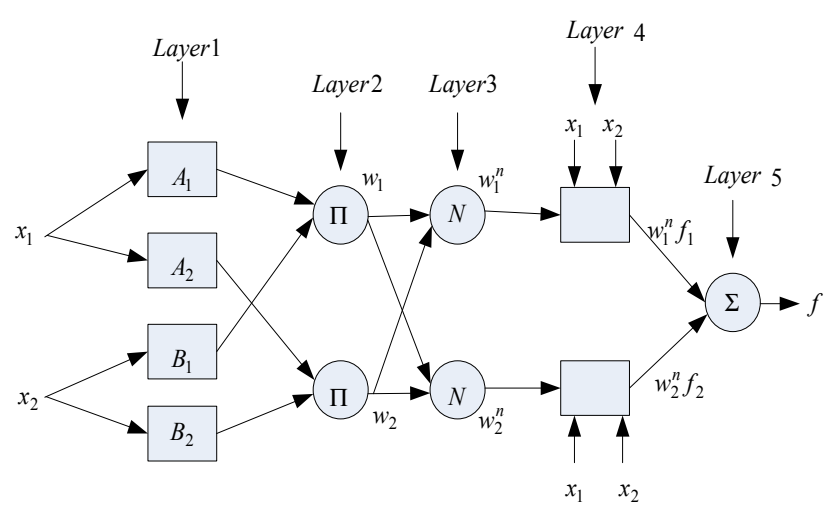

Fig.1. ANFIS structure with 2 inputs and 1 output the ratio of each input derived by total weights.

Layer 5: Single node in this layer is a circle node labeled $\Sigma$ that means for computes overall output as the summation of all incoming signals.

$$
O_{i}^{5}=\sum_{i=1}^{2} \bar{w}_{i} \cdot z_{i}, \quad i=1,2
$$

From Fig.1 overall output can be expressed as linear combinations of the consequent parameters. The output $f$ in Fig. 1 can be rewritten as

$$
\begin{aligned}
& f=\frac{w_{1}}{w_{1}+w_{2}} f_{1}+\frac{w_{2}}{w_{1}+w_{2}} f_{2} \\
& =\bar{w}_{1} f_{1}+\bar{w}_{2} f_{2} \\
& =\left(\bar{w}_{1} x_{1}\right) p_{1}+\left(\bar{w}_{1} x_{1}\right) q_{1}+\left(\bar{w}_{1}\right) r_{1}+\left(\bar{w}_{2} x_{1}\right) p_{2}+\left(\bar{w}_{2} x_{2}\right) q_{2}+\left(\bar{w}_{2}\right) r_{2}
\end{aligned}
$$

In this paper, the membership functions of ANFIS have two parameter inputs error (E) and change of error (DE) and a single output $u$. The inputs and output $\mathrm{E}, \mathrm{DE}$, and $u$ are intuitively partitioned into seven fuzzy linguistic spaces $\{\mathrm{NB}$, NM, NS, Z, PS, PM, PB $\}$ which tabulated in Table I. Fig. 2 and 3 exhibits the form membership function utilized Gaussian type.

\section{B. Self Tuning Regulator}

Basically, Self Tuning Regulator and ANFIS are invoked to accomplish different task, ANFIS is main controller, and STR is controller to improve the performance of ANFIS and keep plant response always within the stability region. Self tuning regulator used in this paper's established from Fuzzy inference system which is intuitively determined from system response. Similar with ANFIS model, STR has two inputs error (E) and change of error (DE) and a single output $u$. Rule base of self tuning regulator from plant output can be derived on Fig. 4. For STR the form membership function of $\mathrm{E}$ and DE employ triangular type which exhibits on Fig 5 and 6.

First of all, Define that the term sets of input/output variables have five membership functions, with terms of negative big (NB), negative small (NS), zero (Z), positive small (PS), and positive big (PB). The prototype of fuzzy control rules is tabulated in Table II. In point $A$ if $E$ is positive and DE is negative then $u$ should be positive. In the point $B, E$ is negative smaller than the point $a$, and $D E$ is positive, so it must be decreased $u$ to avoid excessive overshoot. In the point $\mathrm{c}, \mathrm{E}$ is positive big and $\mathrm{DE}$ is negative big, in order to get steady state, $u$ has to be increased. In the 
point $\mathrm{d}, \mathrm{E}$ is positive small and $\mathrm{DE}$ is negative small to approach steady state value $u$ should be decreased.

TABLE I: RULE BASE OF CONTROLLER ANFIS

\begin{tabular}{|c|c|c|c|c|c|c|c|}
\hline DE\E & NB & NM & NS & $Z$ & PS & PM & PB \\
\hline NB & NB & NB & NB & NB & NM & NS & Z \\
\hline NM & NB & NB & NB & NM & NS & Z & PS \\
\hline NS & NB & NB & NM & NS & Z & PS & PM \\
\hline Z & NB & NM & NS & Z & PS & PM & PB \\
\hline PS & NM & NS & Z & PS & PM & PB & PB \\
\hline PM & NS & Z & PS & PM & PB & PB & PB \\
\hline PB & Z & PS & PM & PB & PB & PB & PB \\
\hline
\end{tabular}

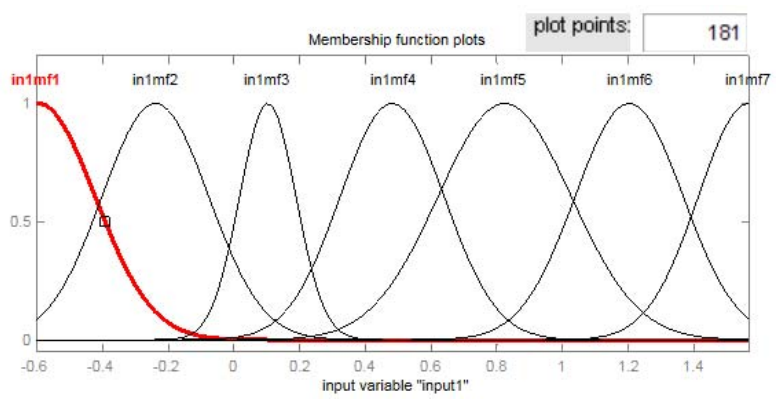

Fig.2. Membership function of error (E) for ANFIS controller

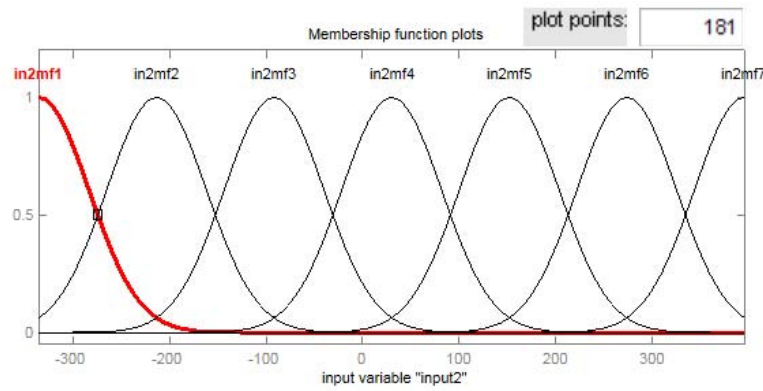

Fig.3. Membership function of derror (DE) for ANFIS controller

\section{Cascade Control (Pressure Rig 38-714)}

Cascade control is one of complex control architecture commonly used in the real word to have better response than single loop architecture. Cascade structure gets many advantages than the conventional structure, expecially for rejecting disturbance in the systems.

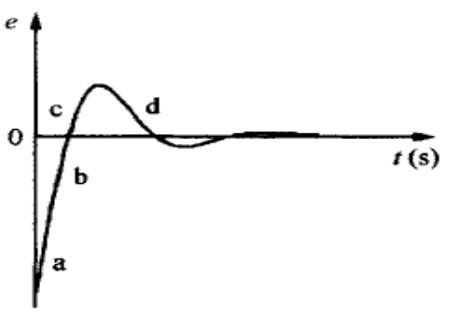

Fig.4. Rule justification by using system step response

TABLE II: RULE BASE OF STR

\begin{tabular}{|l|r|r|r|r|r|}
\hline DElE & NB & NS & \multicolumn{1}{c|}{$Z$} & \multicolumn{1}{c|}{ PS } & PB \\
\hline NB & PB & PB & PB & $Z$ & $Z$ \\
\hline NS & PB & PB & PS & $Z$ & NS \\
\hline$Z$ & PB & PS & $Z$ & NS & NB \\
\hline PS & PS & $Z$ & NS & NB & NB \\
\hline PB & Z & NS & NB & NB & NB \\
\hline
\end{tabular}

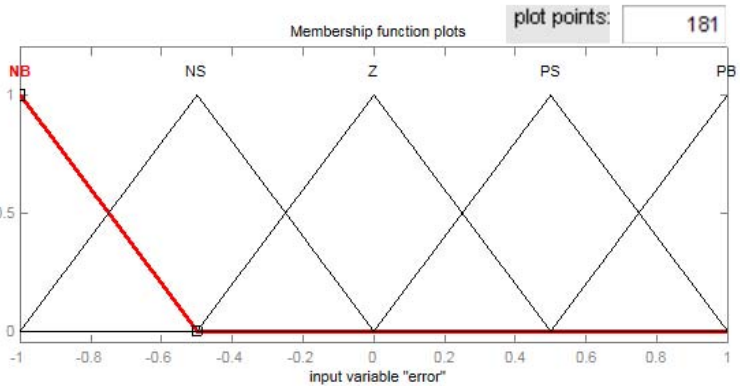

Fig.5. Membership function of error (E) for STR

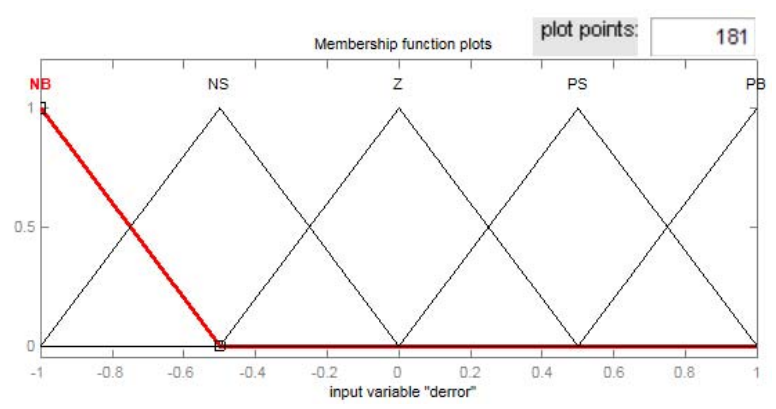

Fig.6. Membership function of derror (DE) for STR

It is reasonable because cascade control has inner loop for fastly compensating disturbance before it is influenced the main control variable.

Plant which used in this paper is pressure rig 38-714. it is able to be configured to represent cascade control architecture in which inner loop and outer loop variables are flow, and pressure. Fig. 7 exhibits a P\&ID diagram of pressure rig 38-714.

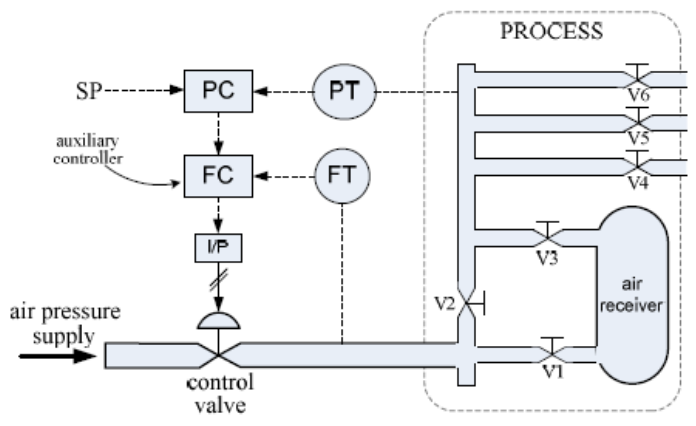

Fig.7. P\&ID diagram of pressure rig 38-714

The way to operation pressure rig 38-714 with given set point from PC and converted to be voltage (0-5 volt). Voltage's changed to be current (4-20 ma), in order to be input of I/P converter. A I/P converter undertakes conversion current to be pressure (3-15 psi). Pressure's detected by pressure sensor via pressure transmitter. Sensor's obtained by differential pressure sensor through flow transmitter. V4, V5, V6, V2, V3, V1 are disturbance of the system which's represented as open or close manual valve.

\section{SySTEM DESIGN}

At this section, the description of pressure rig 38-714's exhausted, modeling of pressure rig 38-714 using ARX's and the function of STR are also clearly discussed.

Identification of pressure rig $38-714$ is achieved by applying random input signal through pseudo random binary sequence method. This method's is termed dynamic identification. The first sets pressure rig 38-714 in nominal 
load in which V4, V5 are opened, and V6 is closed. Set point given between 2-3 volt and bits signal used for pseudo random binary sequence (PRBS) are 1-1-1-0-0-0-1-0-1-0. 1 represent 3 volt and 0 represent 2 volt. Because of inner loop's much faster than inner loop therefore plant may be identified as decouple, it means that outputs are estimated independently. Identification's conducted with open loop configuration. Fig. 8, 9, and 10 show PRBS signal, pressure open loop response, and flow open loop response respectively.

After that, ARX model might be employed to estimate each control variable. Pressure and flow are modeled as one and two ordered system respectively, in which norm error of those are seek the smallest norm error. Equation 8, and 9 are mathematical description of pressure and flow.

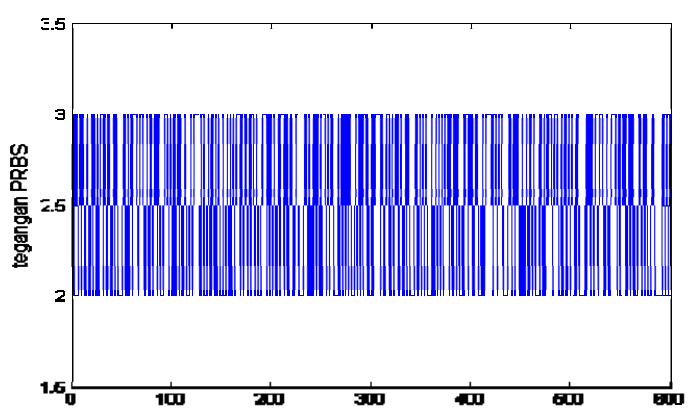

Fig.8. Signal pseudo random binary sequences
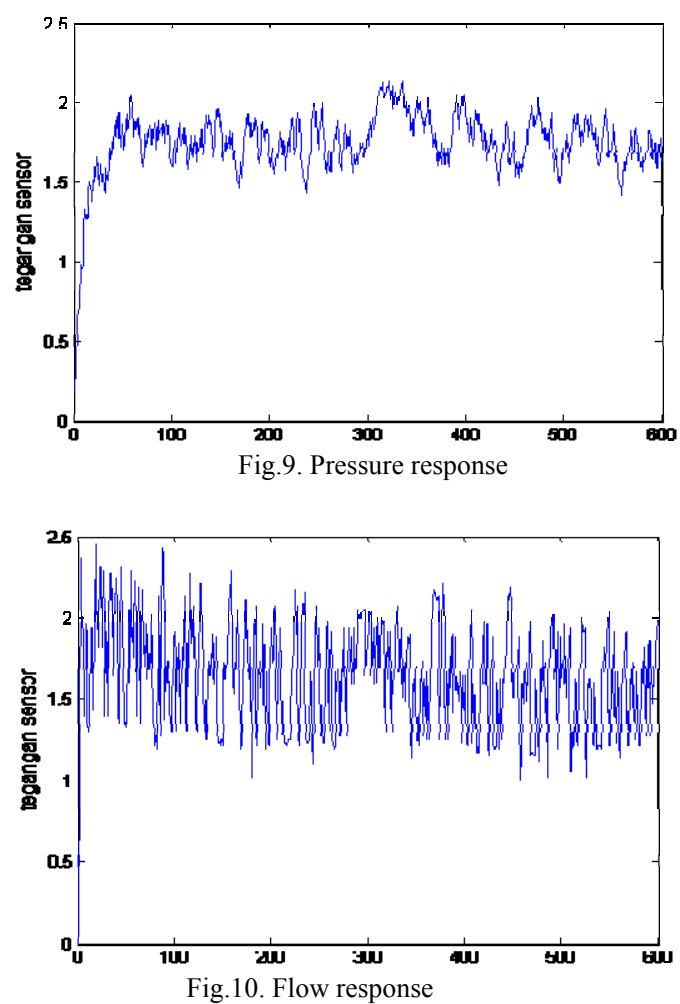

$$
\begin{gathered}
G(s)=\frac{0,8}{180 s+1} \\
G(s)=\frac{12 s+1}{s^{2}+63 s+8,4}
\end{gathered}
$$

The complete system in this paper is added with STR for improving the performance of system. Self tuing regulator can be categorid as adaptive control system. The basic principle of adaptive control system is able to change if the condition is changed dynamically.

\section{Simulation AND Discussion}

This paper compares ANFIS using STR and only ANFIS. The control scheme of the ANFIS with STR and ANFIS are shown in Fig.11 and 12.

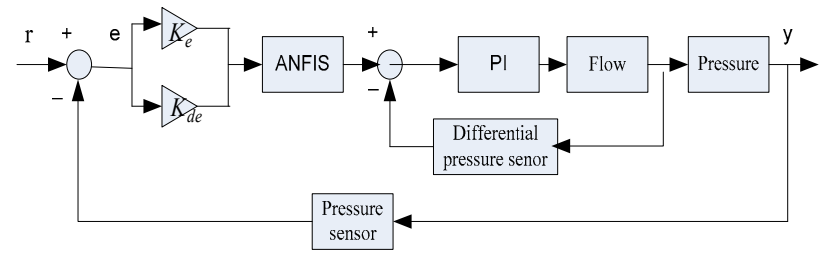

Fig.11. Architecture system with controller ANFIS

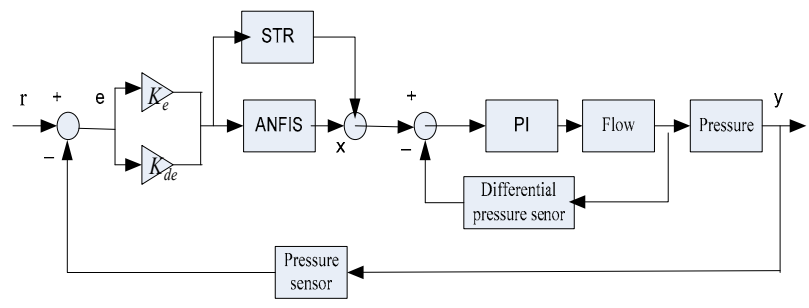

Fig.12. Architecture system with controller ANFIS + STR

The value of $K_{e}$ and $K_{d e}$ are determined 0,5 to satisfy the requirement. Comparion between both algorithm are shown in Fig. 13.

In Table III exhibits the transient responses algorithm ANFIS using STR and ANFIS. Both of algorithms are ejected within the same input step (the value of step input is 2) while $100 \mathrm{~ms}$. It can be seen that ANFIS using STR performs better response than another, it is verified with faster to reach steady state criterion. Compared from transient responses, ANFIS has the value of time rise (tr) $21 \mathrm{~ms}$. In ANFIS using STR is acquired value of $9 \mathrm{~ms}$ for the time rise. Furthermore, for the value of settling time ANFIS using STR gives $12 \mathrm{~ms}$ less than the settling time of ANFIS (29 ms). It is fair to be said that extended approach of ANFIS using STR is able to improve Pure ANFIS.

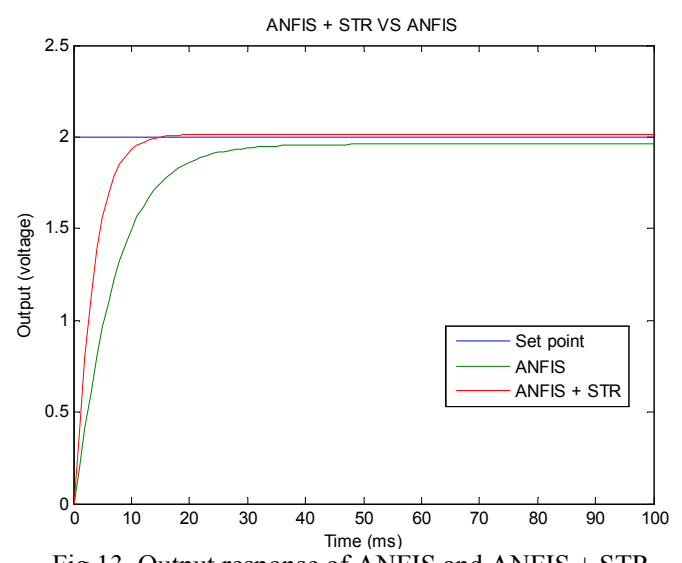

Fig.13. Output response of ANFIS and ANFIS + STR

TABLE III: TRANSIENT PARAMETER RESPONSE

\begin{tabular}{|c|c|c|}
\hline Algorithm Mode & $\operatorname{tr}(10 \%-90 \%)$ & $\operatorname{ts}(5 \%)$ \\
\hline ANFIS & $9 \mathrm{~ms}$ & $12 \mathrm{~ms}$ \\
\hline ANFIS with STR & $21 \mathrm{~ms}$ & $29 \mathrm{~ms}$ \\
\hline
\end{tabular}

\section{CONCLUSION}

Applying extended ANFIS to solve control task gives satisfied results in performance than just using pure ANFIS. 
From experiment, shows that ANFIS using STR can much improve the performance a system, it might be checked from faster increasing of rise time and settling time within transient response. The ANFIS extension of this paper is actually simple, nevertheless it's able to give improvement of the ANFIS. This technique's actually ready to be implemented to solve more complicated control task such as: Networked Control System, Nonlinear Control, etc. this paper actually need to be elaborated which will be given in the future work.

\section{REFERENCES}

[1] Bawane. N, Kothari. A. G, Kothari. D. P, "ANFIS based on HVDC control and fault identification of HVDC converter," HAIT Journal of Science and Engineering B, vol. 2, Issues 5-6, p. 673-689.

[2] T. Mahmoud, T. S, Hong, M. Marhaban,"Neuro fuzzy and self tuning fuzzy controller to improve pitch and yaw control system response of twin rotor MIMO system," World academy of science, 50, 2009.

[3] W. Farag, V. Quintana, G, L, Torres, " A genetic based neuron-fuzzy approach for modeling and control of dynamic systems, "IEEE Trans. Neural Network, vol. 9, no. 5, Sep. 1998.

[4] B. Allaoula, A. Laoufi, B. Gasbaoui, A. Abderahmania, "Neuro fuzzy DC motor speed control using PSO," Leonardo electronic journal of practice and technologies, ISSN, p. 1-18, 2009.

[5] S. Kurnaz, O. Cetin, O. Kaynak, "ANFIs based on outonomous flight control of UAV, “Elsevier, Expert system with applications, 2010.

[6] A. A, Saifizul, Z. Zainon, N. A. A, Osman, C. A, Azlan, U. F. S. U, Ibrahim,"Intelligent control for self erecting pendulum via ANFIS," American journa; of applied science, ISSN, 2006.

[7] S. A, Mazhari, S. Kumar, "Hybrid GA tuned RBF based neuro fuzzy for robotic manipulator," International journal of electrical, computer, and system engineering, 2008.

Mahardhika Pratama was born in Surabaya, Indonesia, 1988. He received B.Eng. from Electrical Engineering Department, Sepuluh Nopember Institute of Technology with First Class Honor. He is currently master student in the Nanyang Technological University, Singapore. In his relatively young ages, he has published 15 papers in the international conferences and journals. Now, he is also working as attachment in Singapore Institute of Manufacturing Technology (SIMTech) within the funded project of A-Star science and engineering research agency Singapore-Poland. His researches interests are in the area of Fuzzy Neural Network (FNN), type 2 fuzzy logic, intelligent control, modeling and identification, nonlinear control, and complex system. Dhika is also member of IEEE computational intelligent society, and control system society,
Indonesian Soft Computing Society, International Association of Computer Science and Engineering Technology (IACSIT).

Samsul Rajab was born in Wapunto, Indonesia in 1987. He received the B.S. degree from Department of electrical Engineering, Sepuluh Nopember Institute of Technology, Surabaya, Indonesia, in 2006. Since 2009, he has been a assistant lecture in the Analog Control System Laboratory at the Sepuluh Nopember Institute of Technology. In 2010 He joint with research group in Control System Laboratory. His research interests in the area of intelligent control with it's application and nonlinear control.

Er Meng Joo Professor Er Meng Joo is currently a Full Professor in Electrical and Electronic Engineering and Director of Renaissance Engineering Programme, College of Engineering (CoE). He is also an elected member of the NTU Advisory Board, the NTU Senate Steering Committee and the Institution of Engineers, Singapore (IES) Council. He has authored three books entitled "Dynamic Fuzzy Neural Networks: Architectures, Algorithms and Applications" and "Engineering Mathematics with Real-World Applications" published by McGraw Hill in 2003 and 2005 respectively, and "Theory and Novel Applications of Machine Learning" published by In-Tech in 2009, 14 book chapters and more than 400 refereed journal and conference papers in his research areas of interest. Professor Er was the winner of the IES Prestigious Publication (Application) Award in 1996 and IES Prestigious Publication (Theory) Award in 2001. He was awarded a Commonwealth Fellowship tenable at University of Strathclyde in 2000. He received the Teacher of the Year Award for the School of EEE in 1999, School of EEE Year 2 Teaching Excellence Award in 2008 and the Most Zealous Professor of the Year Award 2009. He also received the Best Session Presentation Award at the World Congress on Computational Intelligence in 2006. Furthermore, together with his students, he has won more than 30 awards at international and local competitions. Currently, he serves as the Editor-in-Chief for the IES Journal B on Intelligent Devices and Systems, an Area Editor of International Journal of Intelligent Systems Science and an Associate Editor of twelve refereed international journals, namely IEEE Transactions on Fuzzy Systems, International Journal of Fuzzy Systems, Neuro computing, International Journal of Humanoid Robots, Journal of Robotics, International Journal of Mathematical Control Science and Applications, International Journal of Applied Computational Intelligence and Soft Computing, International Journal of Fuzzy and Uncertain Systems, International Journal of Automation and Smart Technology, International Journal of Modeling, Simulation and Scientific Computing, International Journal of Intelligent Information Processing and the Evolving Systems. Furthermore, he serves a Guest Editor of International Journal of Neural Systems and an editorial member of Open Electrical and Electronic Engineering Journal, Birkhauser Autonomous Editorial Board and EE Times-Asia. Professor Er Meng Joo's areas of expertise are intelligent control theory and applications, fuzzy logic and neural networks and robotics and automation. His current works focus on control theory and applications, fuzzy logic and neural networks, computational intelligence, cognitive systems, robotics and automation, sensor networks and biomedical engineering. 